\title{
Janice L. Ristock: Intimate Partner Violence in LGBTQ Lives
}

\author{
Routledge, 2011, 338 pages, $\$ 125.00$
}

\author{
Susan J. Wurtzburg
}

Published online: 27 September 2013

(C) Springer Science+Business Media New York 2013

LGBTQ (lesbian/gay/bisexual/transgender/queer) intimate partner abuse has been neglected by domestic violence academics, although workers at shelters and other intervention agencies recognize its prevalence, and the need for more research and data-informed resources. A caveat to this statement is that there are many studies dealing with lesbian violence (probably because these individuals have presented at womenfocused intervention agencies for decades, so there was greater awareness of them initially), but little research deals with the transgendered and queer communities.

Ordinarily, this lack of research might only be problematic for social science advancement, but in this case, it has clear implications for members of the LGBTQ community, who need help dealing with inter-partner violence. Lack of data translates into uninformed assistance, or no assistance by police, courts, shelters, and other service providers. A typical strategy, given the low number of applicable studies has been to apply knowledge about heterosexual intimate battery in a somewhat haphazard fashion to understanding LGBTQ family violence. This policy has been about as effective a helping tool as attempts from previous decades to use information about white, middle-class families for understanding domestic challenges for people of color.

Ristock and her colleagues, recognizing this need, have compiled an important resource for better understanding LGBTQ violence, with research from Australia, Canada, the United States, and the United Kingdom. While there is excellent geographical spread, there is less attention paid to ethnic diversity, which is an observation, not a criticism, since there are only so many chapters, which can be included in a single

S. J. Wurtzburg $(\bowtie)$

Department of Sociology, University of Hawaii at Manoa,

2424 Maile Way, Rm. 214, Honolulu, HI 96822-2223, USA

e-mail: wurtzbur@hawaii.edu book. A notable exception in this regard is the focus on First Nations' (aboriginal) communities in North America, which is valuable, since indigenous conceptions of gender and sexuality may vary significantly from those identified in nonaboriginal groups.

In addition to highlighting First Nations' community members' diversity of beliefs about violence among Two Spirits (pan-American Indian term, often glossed as LGBTQ, although with pre-European contact legitimacy, and much diversity in expression), a major focus of this book is "complicating" ideas about LGBTQ self-definition, and conceptions of love, appropriate relationships, and violence. The authors encourage researchers, service providers, and activists to move beyond stereotypical notions about the LGBTQ community, and to engage fully with its diversity, in order to provide valuable interventions, with the possibility of true change.

This focus on social change is a major strength of this publication, with the final five chapters dealing with challenging violence, and encouraging healthy relationships and communities. Programs located in Canada, the U. S., and Australia are highlighted, once again with inclusion of aboriginal North American peoples, and with attention paid to rural Australians. People living in remote areas have tremendous difficulty in accessing services and support so this is a really useful section.

Another way in which this publication piqued my interest was the good collaboration between researchers and service providers, which is not always apparent in multi-authored volumes. The chapters are well edited, and each contributes important learning, with consistently high quality writing and reasoning. I think this book could be used effectively in an upper-level undergraduate course about domestic violence, or in a graduate sociology, anthropology, or social work class. The volume is probably more suited for a specialty audience, and will be most appreciated by researchers and program providers. 